\title{
CN ANOMALIES IN THE HALO SYSTEM AND THE ORIGIN OF GLOBULAR CLUSTERS IN THE MILKY WAY
}

\author{
Daniela Carollo ${ }^{1,5}$, Sarah L. Martell ${ }^{2}$, Timothy C. Beers ${ }^{3,6}$, and Ken C. Freeman ${ }^{4}$ \\ ${ }^{1}$ Department of Physics and Astronomy-Astronomy, Astrophysics and Astrophotonic Research Center Macquarie \\ University-North Ryde, 2109 NSW, Australia; daniela.carollo@mq.edu.au \\ 2 Australian Astronomical Observatory, North Ryde, 2109 NSW, Australia; smartell@aao.gov.au \\ ${ }^{3}$ National Optical Astronomy Observatory, Tucson, AZ 85719, USA; beers@ noao.edu \\ ${ }^{4}$ Research School of Astronomy \& Astrophysics, Australian National University \& Mount Stromlo Observatory, \\ Cotter Road, Weston, ACT 2611, Australia; kcf@mso.anu.edu.au \\ Received 2012 December 11; accepted 2013 March 16; published 2013 May 7
}

\begin{abstract}
We explore the kinematics and orbital properties of a sample of red giants in the halo system of the Milky Way that are thought to have formed in globular clusters based on their anomalously strong UV/blue CN bands. The orbital parameters of the $\mathrm{CN}$-strong halo stars are compared to those of the inner- and outer-halo populations as described by Carollo et al., and to the orbital parameters of globular clusters with well-studied Galactic orbits. The $\mathrm{CN}$-strong field stars and the globular clusters both exhibit kinematics and orbital properties similar to the inner-halo population, indicating that stripped or destroyed globular clusters could be a significant source of inner-halo field stars, and suggesting that both the $\mathrm{CN}$-strong stars and the majority of globular clusters are primarily associated with this population.
\end{abstract}

Key words: Galaxy: evolution - Galaxy: formation - Galaxy: halo - Galaxy: structure - globular clusters: general - stars: abundances - surveys

Online-only material: color figures, machine-readable tables

\section{INTRODUCTION}

Although globular clusters (GCs) were once held up as the prototype of simple stellar populations, the presence of multiple stellar populations in globulars is now well recognized. Evidence for this complexity is found in both the elemental abundance distributions of individual cluster stars and from clearly separable multiple sequences in well-measured color-magnitude diagrams. The chemical pattern most useful to identify multiple populations is primarily the abundances of light elements formed by proton-capture nucleosynthesis in the later stages of stellar evolution. Light-element abundance inhomogeneities, such as the $\mathrm{C}-\mathrm{N}, \mathrm{O}-\mathrm{Na}$, and $\mathrm{Mg}$ - $\mathrm{Al}$ anticorrelations, have been found among stars on the red giant branches in essentially all the GCs where sufficient data exists (e.g., Gratton et al. 2001; Ramirez \& Cohen 2002; Kayser et al. 2008; Carretta et al. 2009a, 2009b and references therein; Smolinski et al. 2011b). During the evolution of GCs, a large fraction of stars may have been lost through early violent relaxation following gas expulsion, mass loss from the most massive stars (Baumgardt et al. 2008), and the evaporation of a significant fraction of stars in two-body encounters over long timescales (McLaughlin \& Fall 2008). First-generation stars born in GCs that have migrated into the halo system cannot be readily distinguished from stars born outside of the clusters based on their chemical abundances. The situation differs for secondgeneration stars, because their peculiar chemical compositions are believed to be obtained only as a result of their formation inside the deep gravitational potential well of a GC; this abundance signature acts as a "chemical tag" that provides the opportunity to identify them even after they have been lost to the halo field.

Earlier studies of halo-star chemistry (e.g., Pilachowski et al. 1996; Stephens \& Boesgaard 2002; Gratton et al. 2004; Venn et al. 2004) did not find any of these second-generation

\footnotetext{
5 INAF-Osservatorio Astronomico di Torino, Pino Torinese, Italy.

6 Department of Physics, and Astronomy and JINA: Joint Institute for

Nuclear Astrophysics, Michigan State University, E. Lansing, MI 48824, USA.
}

"migrant" stars, bolstering the idea that only star formation in GCs is able to produce the characteristic light-element abundance anomalies. More recently, second-generation stars ${ }^{7}$ have been identified in the halo field by Martell \& Grebel (2010) and Martell et al. (2011), using medium-resolution spectroscopic data for giants from the Sloan Extension for Galactic Understanding and Exploration (SEGUE; Yanny et al. 2009). The CN anomalies were found among SEGUE giants with low or normal carbon abundances (inferred from the strength of the $\mathrm{CH} G$ band at $4300 \AA$ ) that exhibit unusually strong absorption in the UV/blue $\mathrm{CN}$ band (3883 $\AA$ ), from which it is inferred that they possess high nitrogen abundances. Additional evidence for their presence outside of GCs is provided by Carretta et al. (2010), using a compilation of literature abundance data, and by Ramirez et al. (2012), using high-resolution spectra of the nearby Nissen \& Schuster (2010) sample of halo dwarf stars.

In order to put the $\mathrm{CN}$-anomalous stars found in the halo into their proper context, it is important to recognize that ideas concerning the nature of the halo have evolved over the past few years. For example, Carollo et al. $(2007,2010)$ have argued that the halo of the Milky Way comprises at least two smooth stellar components, the inner and outer halos, possessing different peak metallicities $\left([\mathrm{Fe} / \mathrm{H}]_{\text {inner }} \sim-1.6 ;[\mathrm{Fe} / \mathrm{H}]_{\text {outer }} \sim-2.2\right)$, different spatial distributions, and different kinematics. The inner halo has a flatter density profile than the nearly spherical outer halo, and has almost zero mean rotation, while the outer halo exhibits a significantly retrograde rotation. The transition from dominance by the inner-halo population (IHP) to the outer-halo population (OHP) occurs in the range 15-20 kpc from the Sun.

In this paper, we explore the kinematics and orbital properties of the $\mathrm{CN}$-strong field giants from the sample of Martell et al. (2011), as well as for a subset of GCs with available proper motions, in order to assess whether $\mathrm{CN}$-strong field stars are

\footnotetext{
7 Hereafter, we refer to all later generations of stars in globular cluster as "second-generation," even though they may have been born during distinct bursts of star formation.
} 
better associated with the IHP or OHP, and to infer the likely fate of their parent GCs. This paper is organized as follows. In Section 2, we discuss the origin of the chemical abundance variations in GCs, and the possible connection with the Galactic halo system. Section 3 describes the data and the selection of the field-star sample, together with the derivation of the $\mathrm{CN}$ line strengths. In addition, this section describes the selection of a sample of GCs with available proper motions. In Section 4, we describe the derivation of the kinematics and orbital parameters for the field star and GC samples. Section 5 presents an analysis of these data sets. Our main results are summarized in Section 6, along with a brief discussion of their implications.

\section{GLOBULAR CLUSTERS AND THE GALACTIC HALO}

\subsection{The Origin of Chemical Abundance Variations in Globular Clusters}

The observed light-element abundance variations in GCs are often explained in the context of multiple generations of stars within the clusters. Stars with atypical light-element abundances were likely to have formed from material enriched by the ejecta of earlier stellar generations in the cluster (Gratton et al. 2001, 2004; Ramirez \& Cohen 2002; Carretta et al. 2010). Suggestions for the polluters include asymptotic giant branch stars (Cottrell \& Da Costa 1981; Parmentier et al. 1999; Ventura et al. 2001), rapidly rotating massive stars (Decressin et al. 2007), and massive binary stars undergoing mass transfer (de Mink et al. 2009). First-generation stars in GCs are expected to have been stars with typical Population II compositions (Truran \& Arnett 1971), similar to that of the field halo stars. Typically, one-third to two-thirds of the stars in a given GC are thought to be second- (or later-) generation objects, and exhibit the distinct light-element anomalies (e.g., Kraft 1994; Carretta et al. 2009a).

The relatively high observed ratios of second- to firstgeneration stars in GCs create a significant problem for multiplegeneration models of GC evolution-it is not possible for the present-day first-generation stars to have produced sufficient material to pollute the present-day second generation. A top-heavy initial mass function for the first generation has been suggested as a possible solution to this "mass budget problem" (e.g., Cannon et al. 1998), but most current GC formation models (e.g., D'Ercole et al. 2008, 2010; Conroy 2012) assume that the first generation of stars was initially much more massive (by a factor of 10-20) than it is today. This additional mass at an early point in cluster evolution results in more sources for stellar nucleosynthesis feedback, and also raises the escape velocity, making it more likely for clusters to retain sufficient polluted gas to form a second generation of stars. This immediately implies that many or most of the first-generation stars that initially formed in GCs have been subsequently lost to the halo field populations.

Theoretical models predict that as much as $90 \%-95 \%$ of the first-generation stars have been lost from GCs at relatively early times (D'Ercole et al. 2008; Vesperini et al. 2010, 2013; Conroy 2012). It is not yet clear if the second-generation stars were lost all at once, or during later multiple episodes of GC tidal disruption. Martell et al. (2011) adopt this scenario to predict that $\sim 17 \%$ of the halo field stars (exhibiting both firstand second-generation abundance patterns) were born in GCs, while Schaerer \& Charbonnel (2011) estimate that 5\%-8\% of halo field stars originally formed in GCs.

So far, we have considered the chemical evolution of GCs in a two-generation scenario, in which both generations of star formation went on within the cluster itself. The very young and massive LMC cluster NGC 2070 suggests a possible variation on this scenario. NGC 2070 is a globular-like star cluster with a mass of about $5 \times 10^{5} M_{\odot}$ (Bosch et al. 2009), and an age of only two million years (Massey \& Hunter 1998). The cluster is immersed in the massive 30 Dor nebula, which contains about $4 \times 10^{6} M_{\odot}$ of $\mathrm{H} \mathrm{I}$ (S. Kim 2013, private communication), H II (Kennicutt 1984), and CO (Pineda et al. 2009), plus about 4 $\times 10^{4} M_{\odot}$ of hot gas (Wang 1999). Active star formation is going on around the NGC 2070 cluster; some of the stars have masses $>100 M_{\odot}$ (Massey \& Hunter 1998), and some of the surrounding young stars are older than the cluster itself. This spatially extended and ongoing burst of star formation started before the formation of the cluster, and has pressurized the environment and contributed to its chemical evolution.

Although the metallicity of the 30 Dor region is higher than that of the Galactic halo clusters, the 30 Dor system may be much like the early globular-cluster-forming fragments envisaged by Searle \& Zinn (1978). If this kind of environment is typical of the formation of the halo GCs, then the star formation in an extended region around the cluster may provide the first generation of stars, and the cluster itself is the second generation.

The first generation of stars forms from the background interstellar medium (ISM), and is loosely bound to the cluster. Eventually most of them will escape into the halo. The second generation (the cluster stars) forms partly from the background ISM and partly from infalling gas that has been further enriched by the evolution of the massive stars of the surrounding first generation. Although these second-generation stars are initially bound to the cluster, some will escape into the halo during the dynamical evolution of the cluster and may be recognized as the Martell et al. halo stars with $\mathrm{CN}$ anomalies.

In this scenario, the mass of the first generation need not be tightly related to the mass of the cluster itself, so the ratio of first-generation to second-generation stars escaping into the halo is likely to vary from cluster to cluster. In particular, the mass of first-generation stars now within the cluster is not required to be sufficiently large to produce the fusionprocessed material leading to the abundance offsets seen in the second generation. Furthermore, the ongoing star formation in the region surrounding the cluster will also produce stars with $\mathrm{CN}$ anomalies, as its star formation continues and it evolves chemically. These $\mathrm{CN}$-enhanced stars would escape and contribute to the halo's population of anomalous stars. The low total number of such stars observed in the halo puts a limit on the total number of $\mathrm{CN}$-enhanced stars that have come into the halo, either as escapees from the clusters or from their surrounding regions.

Which (if either) of these two enrichment scenarios pertains remains uncertain. A comparison of the properties of the $\mathrm{CN}$ strong stars in the clusters and in the Galactic halo system may provide a useful guide.

\subsection{Possible Connections with the Halo System of the Galaxy}

Carollo et al. (2010) demonstrated that the flattened IHP is essentially non-rotating, with $V_{\phi}=7 \pm 4 \mathrm{~km} \mathrm{~s}^{-1}$, while the near-spherical OHP exhibits a significant retrograde signature, with $V_{\phi} \sim-80 \mathrm{~km} \mathrm{~s}^{-1}$ (where $V_{\phi}$ is the Galactocentric rotational velocity). The velocity ellipsoids of these populations differ as well, such that $\left(\sigma_{V_{R}}, \sigma_{V_{\phi}}, \sigma_{V_{Z}}\right)=(150,95,85) \mathrm{km} \mathrm{s}^{-1}$ for the inner halo and $(159,165,116) \mathrm{km} \mathrm{s}^{-1}$ for the outer halo, evaluated in a Galactocentric cylindrical reference frame. Kinman et al. (2012) presented similar results, based on samples 
of RR Lyrae stars chosen without kinematic bias (a transition from a flattened, essentially non-rotating inner halo to a retrograde spherical outer halo beyond about $12.5 \mathrm{kpc}$ ). Hattori et al. (2013) have used blue horizontal-branch (BHB) stars with available metallicities and radial velocities from the Sloan Digital Sky Survey (SDSS; Gunn et al. 2006; York et al. 2000) in order to demonstrate that the mean rotational velocity of the very metal-poor $([\mathrm{Fe} / \mathrm{H}]<-2.0)$ BHB stars significantly lags behind that of the relatively more metal-rich $([\mathrm{Fe} / \mathrm{H}]>-2.0)$ BHB stars. Furthermore, the relatively more metal-rich BHB stars are dominated by stars with eccentric orbits, while the very metal-poor BHB stars are dominated by stars on rounder, lower-eccentricity orbits. Similar results are reported by Kafle et al. (2013). All of these results are consistent with dual halo described by Carollo et al. (2007, 2010).

Carollo et al. (2012) have used the dual halo paradigm to account for the well-known observed increase of the frequency of carbon-enhanced metal-poor (CEMP) stars with decreasing metallicity (see Beers \& Christlieb 2005 and references therein), as well as for the increase of the CEMP frequency with distance from the Galactic plane (Frebel et al. 2006). Beers et al. (2012) offer additional lines of evidence for the existence of the dual halo. Most recently, An et al. (2013) have used photometric estimates of stellar metallicity for stars in SDSS Stripe 82, along with available proper motions, to argue that even in the relatively nearby volume (5-8 kpc from the Sun), the observed metallicity distribution function (MDF; coupled with the kinematics) of the halo is incompatible with a single population of stars. Chemical, kinematic, and spatial signatures for a dual halo have also been recently found in high-resolution numerical simulations of Milky-Way-like galaxies incorporating baryons (e.g., Zolotov et a. 2010; Font et al. 2011; McCarthy et al. 2012; Tissera et al. 2012).

Tissera et al. (2013) point out that an important distinction should be made between the IHP and the inner-halo region (IHR), as well as between the OHP and the outer-halo region (OHR). Based on the results of Carollo et al., the IHP of the Milky Way possesses an MDF peaked at $[\mathrm{Fe} / \mathrm{H}] \sim-1.6$, extending toward both higher and lower metallicities, including significant numbers of stars at very low metallicity, $[\mathrm{Fe} / \mathrm{H}]<$ -2.0 . The IHR of the Milky Way is located between 5 and 15-20 kpc, where the IHP is the dominant contributor of stars in the metallicity range $-2.0<[\mathrm{Fe} / \mathrm{H}]<-1.0$. Due to the strong metallicity segregation between the inner and outer halos, most of the stars in the IHR with metallicity below $\sim-2.0$ belong to the OHP. The OHP of the Milky Way has an MDF peaked at $[\mathrm{Fe} / \mathrm{H}] \sim-2.2$, extending toward both higher and lower metallicities, including stars with $[\mathrm{Fe} / \mathrm{H}]>-2.0$. The OHR of the Milky Way is located beyond $\sim 20 \mathrm{kpc}$, where the OHP dominates in the low-metallicity regime, $[\mathrm{Fe} / \mathrm{H}]<-2.0$. The majority of the stars at higher metallicity and located in the OHR likely belong to the overlapping IHP, or are members of bound substructures, such as streams, which are not members of the diffuse stellar component. In this context, the distinction between inner- and outer-halo objects (stars or GCs), based solely on their Galactocentric distance or metallicity, has to be reconsidered. An object at Galactocentric distance beyond $20 \mathrm{kpc}$ is located in the OHR, but it well be a member of the IHP.

\section{SELECTION OF THE SAMPLES OF CN-STRONG FIELD STARS AND GLOBULAR CLUSTERS}

The Martell \& Grebel (2010) and Martell et al. (2011) studies of halo field giants drew their data from the
SDSS-II/SEGUE-1 (Abazajian et al. 2009; Yanny et al. 2009) and SDSS-III/SEGUE-2 (Aihara et al. 2011; Eisenstein et al. 2011; C. M. Rockosi et al., in preparation) surveys, respectively. Both SEGUE surveys were spectroscopic extensions of SDSS, with the goal of acquiring broad wavelength-coverage, moderate-resolution $(R \simeq 2000)$ optical spectra of stars in specific Galactic populations. A few examples of those populations are G- and K-disk dwarfs (e.g., Lee et al. 2011b; Cheng et al. 2012; Schlesinger et al. 2012), white dwarf-main-sequence binaries (Rebassa-Mansergas et al. 2012), and distant halo BHB stars (e.g., Xue et al. 2011). To make the spectra readily useful to the broader community, the SEGUE Stellar Parameter Pipeline (SSPP) was developed to estimate metallicities, effective temperatures, surface gravities, and radial velocities for all stars observed as part of SDSS/SEGUE. The SSPP uses a variety of methods, including photometric calibrations, template matching, and spectral indices; details can be found in Lee et al. (2008a, 2008b, 2011a), Allende Prieto et al. (2008), and Smolinski et al. (2011a).

The sample selection for the Martell \& Grebel (2010) and Martell et al. (2011) studies is described thoroughly in those papers, and briefly summarized here. A generous initial selection was made based on SSPP-derived parameters: $\log (g) \leqslant 3.0$, $[\mathrm{Fe} / \mathrm{H}] \leqslant-1.0,(g-r)_{0} \geqslant 0.2, \sigma_{\log (g)} \leqslant 0.5, \sigma_{[\mathrm{Fe} / \mathrm{H}]} \leqslant 0.5$, and a mean signal-to-noise ratio $(\mathrm{S} / \mathrm{N})$ per pixel of 20 or greater. That initial set was then reduced to include only likely red giant branch stars by dividing it into $0.2 \mathrm{dex}$ bins in $[\mathrm{Fe} / \mathrm{H}]$, and rejecting all stars further than $3 \sigma$ in $(g-r)_{0}$ color from the mean red giant branch color-magnitude sequence in that bin. The $\mathrm{S} / \mathrm{N}$ requirement was augmented to ensure high-quality data in the blue spectral features central to their investigation, requiring that the mean $\mathrm{S} / \mathrm{N}$ per pixel in the wavelength range $4000 \leqslant \lambda \leqslant 4100$ be at least 15 . Carbon-enhanced stars were removed from the sample based on the $\mathrm{C}_{2}$ indices defined in Martell \& Grebel (2010). Stars with $[\mathrm{Fe} / \mathrm{H}] \leqslant-1.8$ were also removed, because the $\mathrm{CN}$ and $\mathrm{CH}$ bands become quite weak at low metallicity (see, e.g., Shetrone et al. 2010 and Smolinski et al. $2011 \mathrm{~b}$ for examples of the limits on band-strength analysis in low-metallicity GCs). We have selected those stars from the Martell et al. (2011) sample that have available proper motions, which means that the star satisfies additional criteria designed to eliminate spurious reported motions (see Munn et al. 2004). ${ }^{8}$ Also, stars belonging to the SDSS/SEGUE fields that fall in the direction of the Sagittarius stream were removed in order to excise possible contaminants. After these selections, the remaining number of stars is $N_{\mathrm{Tot}}=1583$; there are $N_{\mathrm{CN}}=42$ among these stars with strong $\mathrm{CN}$ features.

We have also selected a sample of Galactic GCs with available proper motions from the literature, ${ }^{9}$ in order to compare the properties of these GCs with those of the CN-strong stars, and discuss them in the context of the IHP and OHP. The sample comprises 59 GCs for which positions, absolute proper motions, distances, and radial velocities are listed. In this compilation, the locations of the clusters, distances from the Sun, radial velocities, and metallicities are taken from the Harris (1996) database $\left(2010\right.$ update $\left.^{10}\right)$. Errors in the distances are taken to be $10 \%$ of the stated distance. The absolute proper motions are with respect to distant galaxies, QSOs, and/or millisecond pulsars, and are on the ICRS system with respect to the Hipparcos

\footnotetext{
8 Note that all proper motions have been corrected for the systematic error described by Munn et al. (2008).

9 http://www.astro.yale.edu/dana/gc.html

$10 \mathrm{http}: / /$ www.physics.mcmaster.ca/ harris/mwgc.dat
} 
system (in some cases Tycho-2), or with respect to a kinematic model of the Galaxy. See the website describing this effort for more details. The average error in the proper motions is $\sim 1$ mas $\mathrm{yr}^{-1}$. In our analysis, we have removed the GCs in the direction of the Sagittarius and Canis Major dwarf galaxies, in particular: Pal 12 (Irwin 1999; Palma et al. 2002; Bellazzini et al. 2003; Cohen 2004; Carretta et al. 2010; Law \& Majewski 2010), NGC 4147 (Bellazzini et al. 2003; Carretta et al. 2010; Law \& Majewski 2010), and NGC 4590 (Dinescu et al. 1999; Palma et al. 2002; Casetti-Dinescu et al. 2010; Forbes \& Bridges 2010; ${ }^{11}$ Dalessandro et al. 2012). NGC 5466 (Palma et al. 2002; Bellazzini et al. 2003) is likely associated with Sagittarius, while NGC 1851, NGC 1904, NGC 2298, and NGC 2808 are clusters likely associated with Canis Major (Forbes \& Bridges 2010). With such a selection, the final sample comprises $51 \mathrm{GCs}$ (referred to below as $\mathrm{GC}_{\mathrm{PM}}$ ). The kinematics of the remaining GCs for which proper motions are not available are considered below in the context of a Frenk \& White (1980) analysis. From the Harris database we have removed the GCs in the direction of Sagittarius and Canis Major, and those with Galactocentric distance $>50 \mathrm{kpc}$, which may be associated with other dwarf galaxies. This subsample contains 78 GCs.

\section{ANALYSIS}

\subsection{Derivation of Stellar Parameters}

Estimates of $T_{\text {eff }}, \log g$, and [Fe/H] for the field stars in our sample were obtained from the most recent version of the SSPP; typical internal errors are $\sigma_{T_{\text {eff }}} \sim 125 \mathrm{~K}, \sigma_{\log g} \sim 0.25 \mathrm{dex}$, and $\sigma_{[\mathrm{Fe} / \mathrm{H}]} \sim 0.20$ dex. The external errors in these determinations are of a similar size. Due to recent updates of the SSPP, the metallicities of some of the stars in our present sample differ slightly from those used by Martell et al. (2011), which were taken from the seventh and eighth SDSS data releases (DR7, Abazajian et al. 2009; DR8, Aihara et al. 2011), so that the present data set includes a handful of stars with metallicities outside the original range of $-1.8 \leqslant[\mathrm{Fe} / \mathrm{H}] \leqslant-1.0$. The $\mathrm{CN}$ and CH-band strengths in Martell \& Grebel (2010) and Martell et al. (2011), which we have adopted here, were measured using the indices $S(3839)$ and $S(\mathrm{CH})$, defined in Norris et al. (1981) and Martell et al. (2008), respectively. These indices measure the magnitude difference between the integrated flux in a region of spectrum containing the feature of interest and the integrated flux in a nearby region of spectrum unaffected by the feature of interest, in the sense that stronger absorption in the feature produces a larger band strength. The differential index $\delta S(3839)$ was calculated following the method of Norris et al. (1981) by fitting a straight line to the $\mathrm{CN}$-normal stars in the $S(3839)$ versus absolute magnitude plane, and taking the difference between the measured band strength and that line at fixed magnitude.

Distances have been adopted using the approach described by Martell et al. (2011). As described in that paper, heliocentric distances were calculated by a straightforward photometric parallax method from the observed SDSS $(g-r)_{0}$ colors (where reddening corrections were applied from Schlegel et al. 1998), and interpolating within the $12 \mathrm{Gyr}$ Dartmouth isochrones (Dotter et al. 2008) of appropriate metallicity to each star's color to find its absolute $r$ magnitude, then converting the resulting $\left(r-M_{r}\right)_{0}$ distance modulus into a distance. Monte Carlo sampling of the errors on $(g-r)_{0}$ was used to estimate errors

\footnotetext{
11 In this paper, NGC 4590 is argued to be associated with the Canis Major dwarf galaxy.
}

on the resulting heliocentric distances, typically on the order of $10 \%-15 \%$. Galactocentric distances were determined geometrically using the IDL routine $l b d 2 x y z$, available through Goddard Space Flight Center's online IDL Astronomy Library. ${ }^{12}$

\subsection{Derivation of Space Motions and Orbital Parameters}

Proper motions, used in combination with distance estimates and radial velocities, provide the information required to calculate the full space motions (the components of which are referred to as $U, V, W$ ) of our program stars with respect to the local standard of rest (LSR; defined as a frame in which the mean space motions of the stars in the solar neighborhood average to zero). The velocity component $U$ is taken to be positive in the direction toward the Galactic anticenter, the $V$ component is positive in the direction of Galactic rotation, and the $W$ component is positive toward the North Galactic Pole. Corrections for the motion of the Sun with respect to the LSR are applied during the course of the calculation of the full space motions; here we adopt the values $(U, V, W)=(-9,12,7) \mathrm{km} \mathrm{s}^{-1}$ (Mihalas \& Binney 1981). For the purpose of our analysis it is also convenient to obtain the rotational component of a star's motion about the Galactic center in a cylindrical frame; this is denoted as $V_{\phi}$, and is calculated assuming that the LSR is on a circular orbit with a value of $220 \mathrm{~km} \mathrm{~s}^{-1}$ (Kerr \& Lynden-Bell $1986)$. It is worth noting that our assumed values of $R_{\odot}(8.5 \mathrm{kpc})$ and the circular velocity of the LSR are both consistent with two recent independent determinations of these quantities by Ghez et al. (2008) and Koposov et al. (2009). Bovy et al. (2012) have recently determined, on the basis of accurate radial velocities for stars in the APOGEE sub-survey of SDSS-III, that the circular velocity of the LSR is close to $220 \mathrm{~km} \mathrm{~s}^{-1}$.

The orbital parameters of the stars, such as the perigalactic distance (the closest approach of an orbit to the Galactic center), $r_{\text {peri }}$, and apogalactic distance (the farthest extent of an orbit from the Galactic center), $r_{\text {apo }}$, of each stellar orbit, the orbital eccentricity, $e$, defined as $e=\left(r_{\text {apo }}-r_{\text {peri }}\right) /\left(r_{\text {apo }}+r_{\text {peri }}\right)$, as well as $Z_{\max }$ (the maximum distance of a stellar orbit above or below the Galactic plane), are derived by adopting an analytic Stäckeltype gravitational potential (which consists of a flattened, oblate disk, and a nearly spherical massive dark matter halo; see the description given by Chiba \& Beers 2000, Appendix A). Typical errors on the orbital parameters (at $Z_{\max }<50 \mathrm{kpc}$; Carollo et al. 2010) are $\sigma_{r_{\text {peri }}} \sim 1 \mathrm{kpc}, \sigma_{r_{\text {apo }}} \sim 2 \mathrm{kpc}, \sigma_{\text {ecc }} \sim 0.1, \sigma_{Z_{\max }} \sim$ $1 \mathrm{kpc}$. These same methods have been applied to the sample of GCs with available proper motions. Proper motions, distances, and radial velocities for the stars in the Martell et al. (2011) subsample are listed in Table 1, while the derived space motions and orbital parameters are listed in Table 2. An analysis of the kinematics of the sample of GCs, including those without available proper motions, is reported below.

\section{RESULTS}

\subsection{Halo Field Stars}

The left-hand column of panels in Figure 1 shows the index $\delta S$ (3839) for the CN-normal stars (black dots) and CN-strong stars (red dots), as a function of Galactocentric distance, $r$ (top panel), and as a function of the vertical distance, $|z|$ (bottom panel). Note that the sample is limited at small distances by the SDSS/SEGUE bright limit of $g \sim 14$, and at large distances by the requirement that the typical $\mathrm{S} / \mathrm{N}$ per pixel in the blue

\footnotetext{
$\overline{12 \text { http://idlastro.gsfc.nasa.gov/idllibsrch.html }}$
} 

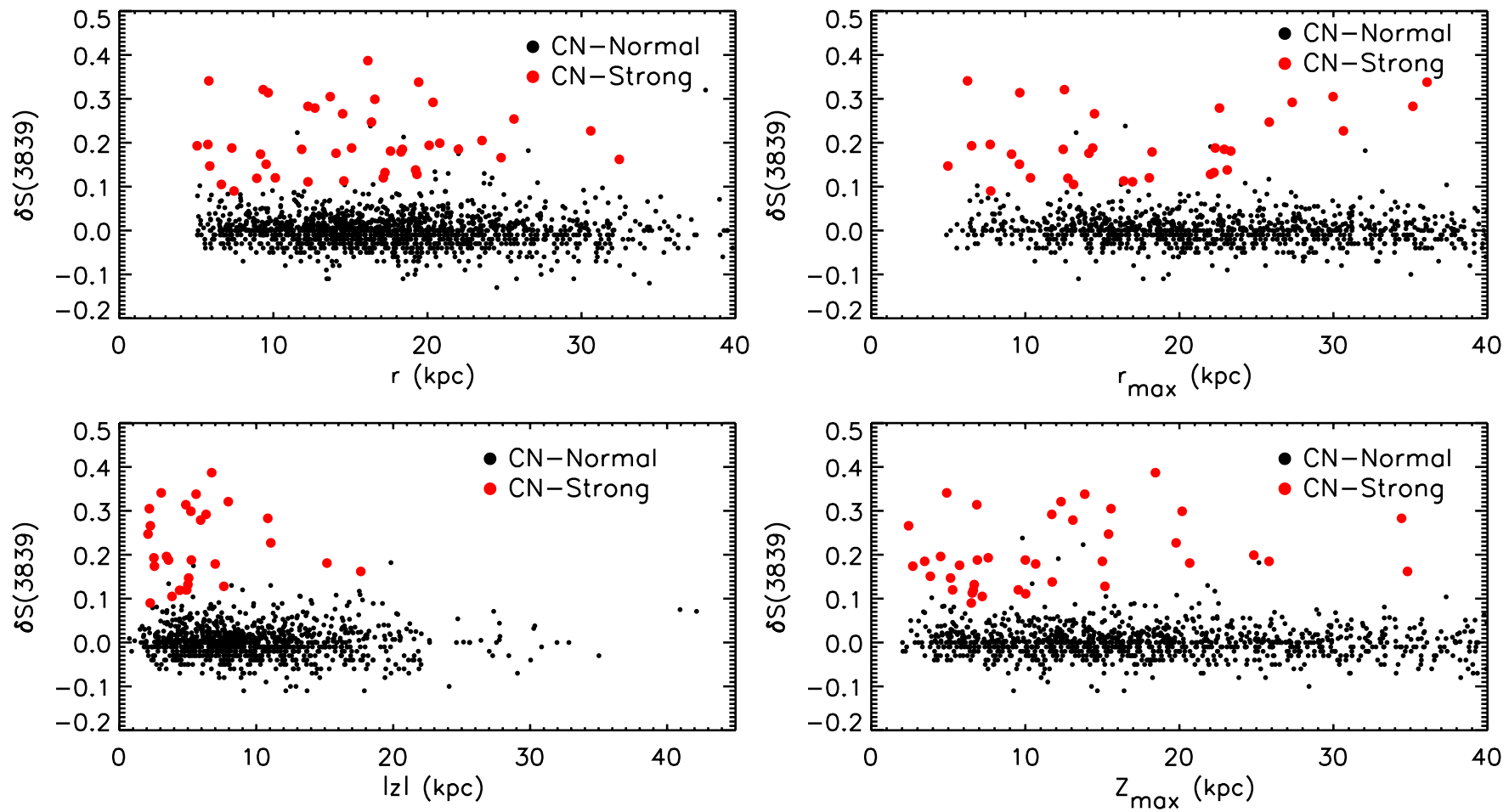

Figure 1. Top-left panel: $\delta S(3839)$ index as a function of the Galactocentric distance, $r$. Black dots represent the CN-normal stars selected from the Martell et al. (2011) sample, while the red dots indicate $\mathrm{CN}$-strong stars. Bottom-left panel: $\delta S(3839)$ index as a function of the vertical distance, $|z|$. Top-right panel: $\delta S(3839)$ index, as a function of apogalactic distance, $r_{\max }$, for the $\mathrm{CN}$-normal stars (black dots) and $\mathrm{CN}$-strong stars (red dots). Bottom-right panel: $\delta S(3839)$ index, as a function of the distance $Z_{\max }$ (the maximum distance of a stellar orbit above or below the Galactic plane), for the CN-normal stars (black dots) and the $\mathrm{CN}$-strong stars (red dots).

(A color version of this figure is available in the online journal.)

Table 1

Parameters for the Martell et al. (2011) Sample

\begin{tabular}{|c|c|c|c|c|c|c|c|c|c|c|c|}
\hline Name & $\begin{array}{c}d \\
(\mathrm{kpc})\end{array}$ & $\begin{array}{c}r \\
(\mathrm{kpc})\end{array}$ & $\begin{array}{c}\mathrm{PM}_{\mathrm{RA}} \\
\left(\mathrm{mas} \mathrm{yr}^{-1}\right)\end{array}$ & $\begin{array}{c}e_{\mathrm{PM}_{\mathrm{RA}}} \\
\left(\operatorname{mas~yr}^{-1}\right)\end{array}$ & $\begin{array}{c}\mathrm{PM}_{\mathrm{DE}} \\
\left(\mathrm{mas} \mathrm{yr}^{-1}\right)\end{array}$ & $\begin{array}{c}e_{\mathrm{PM}} \\
\left(\operatorname{mase}_{\mathrm{DE}} \mathrm{yr}^{-1}\right)\end{array}$ & $\begin{array}{c}V_{\mathrm{RAD}} \\
\left(\mathrm{km} \mathrm{s}^{-1}\right)\end{array}$ & $\begin{array}{c}e V_{\mathrm{RAD}} \\
\left(\mathrm{km} \mathrm{s}^{-1}\right)\end{array}$ & {$[\mathrm{Fe} / \mathrm{H}]$} & $\delta S(3839)$ & $\mathrm{C} \Gamma$ \\
\hline J025155.2-005526.4 & 17.1 & 23.4 & 2.23 & 2.80 & -3.88 & 2.80 & -54.5 & 1.1 & -1.32 & -0.03 & 0 \\
\hline J025141.7-003802.4 & 13.8 & 20.3 & 3.98 & 2.72 & -3.98 & 2.72 & -150.6 & 1.2 & -0.97 & 0.09 & 0 \\
\hline J024954.9-001716.8 & 27.2 & 33.2 & 1.38 & 2.65 & -3.43 & 2.65 & -166.0 & 1.4 & -1.93 & 0.00 & 0 \\
\hline J024958.0-000003.6 & 40.9 & 46.7 & 0.61 & 3.31 & -0.58 & 3.31 & -120.9 & 2.3 & -1.55 & -0.03 & 0 \\
\hline J024959.7-001525.2 & 16.5 & 22.8 & 6.90 & 2.73 & -3.14 & 2.73 & 39.8 & 1.7 & -1.17 & 0.00 & 0 \\
\hline J024536.2-000636.0 & 20.4 & 26.5 & -4.22 & 3.01 & -2.82 & 3.01 & -152.9 & 1.5 & -1.23 & -0.06 & 0 \\
\hline J024511.0-002031.2 & 5.8 & 12.9 & 9.05 & 2.61 & -3.99 & 2.61 & 272.9 & 1.0 & -1.76 & 0.02 & 0 \\
\hline
\end{tabular}

Note. The last column indicates whether the star is considered $\mathrm{CN}$ normal (0) or $\mathrm{CN}$ strong (1).

(This table is available in its entirety in a machine-readable form in the online journal. A portion is shown here for guidance regarding its form and content.)

Table 2

Kinematic and Orbital Parameters for the Martell et al. (2011) Sample

\begin{tabular}{|c|c|c|c|c|c|c|c|c|c|c|c|}
\hline Name & $\begin{array}{c}U \\
\left(\mathrm{~km} \mathrm{~s}^{-1}\right)\end{array}$ & $\begin{array}{c}e_{U} \\
\left(\mathrm{~km} \mathrm{~s}^{-1}\right)\end{array}$ & $\begin{array}{c}V \\
\left(\mathrm{~km} \mathrm{~s}^{-1}\right)\end{array}$ & $\begin{array}{c}e_{V} \\
\left(\mathrm{~km} \mathrm{~s}^{-1}\right)\end{array}$ & $\begin{array}{c}W \\
\left(\mathrm{~km} \mathrm{~s}^{-1}\right)\end{array}$ & $\begin{array}{c}e_{W} \\
\left(\mathrm{~km} \mathrm{~s}^{-1}\right)\end{array}$ & $\begin{array}{c}V_{\phi} \\
\left(\mathrm{km} \mathrm{s}^{-1}\right)\end{array}$ & $\begin{array}{c}e_{V_{\phi}} \\
\left(\mathrm{km} \mathrm{s}^{-1}\right)\end{array}$ & $\begin{array}{l}r_{\max } \\
(\mathrm{kpc})\end{array}$ & $\begin{array}{l}r_{\min } \\
(\mathrm{kpc})\end{array}$ & $\begin{array}{l}Z_{\max } \\
(\mathrm{kpc})\end{array}$ \\
\hline J025155.2-005526.4 & -89.9 & 176.8 & -345.1 & 237.5 & -9.4 & 143.3 & -121.3 & 237.4 & 10.0 & 25.4 & 16.9 \\
\hline J025141.7-003802.4 & -74.9 & 138.9 & -363.7 & 193.0 & 123.2 & 112.8 & -140.5 & 193.1 & 9.4 & 27.3 & 16.8 \\
\hline J024814.1-004106.0 & -442.7 & 457.8 & -438.5 & 578.3 & -401.6 & 364.8 & -185.7 & 578.0 & $\ldots$ & $\ldots$ & $\ldots$ \\
\hline J024954.9-001716.8 & -220.7 & 266.8 & -445.6 & 352.6 & 14.1 & 216.9 & -211.0 & 352.5 & 22.5 & 84.2 & 63.8 \\
\hline J024958.0-000003.6 & -68.7 & 498.8 & -158.8 & 642.2 & 100.5 & 407.2 & 66.2 & 643.4 & 8.1 & 54.2 & 40.0 \\
\hline J024959.7-001525.2 & 224.1 & 171.8 & -528.0 & 239.9 & 102.4 & 137.6 & -319.4 & 241.5 & 21.3 & 107.2 & 75.8 \\
\hline J024850.8-002830.0 & -390.2 & 338.0 & -313.2 & 429.4 & -155.9 & 271.7 & -67.4 & 429.3 & $\ldots$ & $\ldots$ & $\ldots$ \\
\hline J024625.2-005436.0 & -28.1 & 80.5 & -249.6 & 113.6 & 76.3 & 62.6 & -28.6 & 113.7 & 1.4 & 15.1 & 9.8 \\
\hline J024536.2-000636.0 & -487.8 & 241.5 & 65.7 & 290.7 & -170.5 & 190.7 & 320.2 & 291.8 & $\ldots$ & $\ldots$ & $\ldots$ \\
\hline J024511.0-002031.2 & 259.4 & 60.1 & -214.1 & 86.9 & -151.4 & 45.9 & -3.2 & 88.1 & 0.1 & 33.0 & 16.2 \\
\hline
\end{tabular}

(This table is available in its entirety in a machine-readable form in the online journal. A portion is shown here for guidance regarding its form and content.) 
(faint) end of the spectra be at least 15 . These selection effects on the bright and faint ends of the data set operate equally on $\mathrm{CN}$-strong and $\mathrm{CN}$-normal stars, so that the ratio of the two is not affected (see also Section 3.3 of Martell et al. 2011). Note that the $\mathrm{CN}$-strong stars are concentrated in the IHR, and their frequency drops rapidly beyond $20 \mathrm{kpc}$, as previously pointed out by Martell et al. (2011). The distribution of CN-normal and CN-strong stars as a function of the vertical distance $|z|$ shows that most of the $\mathrm{CN}$-strong stars are located in the region $1 \mathrm{kpc}<|z|<8 \mathrm{kpc}$.

The right-hand column of panels in Figure 1 shows the index $\delta S$ (3839) for the $\mathrm{CN}$-normal stars (black dots) and $\mathrm{CN}$-strong stars (red dots), as a function of the maximum Galactocentric distance achieved by stars during their orbits, $r_{\max }$ (top panel), and as a function of the maximum vertical distance achieved by stars during their orbits, $Z_{\max }$ (bottom panel). In Carollo et al. (2007), it was noted that most stars of the IHP do not possess orbits that take them beyond 15-20 kpc. In contrast, stars of the OHP can reach distances well beyond $20 \mathrm{kpc}$ in their orbits. ${ }^{13}$ Similarly, the $\mathrm{CN}$-strong stars in the top-right panel of Figure 1 exhibit apogalactic distances that are mostly located between $5 \mathrm{kpc}$ and $20 \mathrm{kpc}$, and few orbits beyond $20 \mathrm{kpc}$, in agreement with the behavior of the stars of the IHP. Another remarkable feature is that the great majority of the orbits of the $\mathrm{CN}$-strong stars are located within $\mathrm{Z}_{\max }<15 \mathrm{kpc}$, again corresponding to the IHR, where the IHP dominates in the metallicity range -2.0 $<[\mathrm{Fe} / \mathrm{H}]<-1.5$. We have verified that the distributions of $\mathrm{CN}$-normal and $\mathrm{CN}$-strong stars as a function of $r$ or $|z|$ do not change when the entire data set of Martell et al. is considered, including the stars without available proper motions.

Figure 2 shows the derived $V_{R}, V_{\phi}, V_{Z}$ velocity components in the Galactocentric cylindrical reference frame, as a function of metallicity, for the selected samples of $\mathrm{CN}$-normal stars (black dots) and $\mathrm{CN}$-strong stars (red dots). As can be appreciated from inspection of the middle panel, the $\mathrm{CN}$-strong stars at higher metallicity $([\mathrm{Fe} / \mathrm{H}]>-1.1)$ exhibit highly prograde rotational velocities, consistent with that expected for members of the thick-disk and metal-weak thick-disk (MWTD) components, $\left\langle V_{\phi}\right\rangle=185 \mathrm{~km} \mathrm{~s}^{-1}$ and $\left\langle V_{\phi}\right\rangle=125 \mathrm{~km} \mathrm{~s}^{-1}$, respectively (Carollo et al. 2010). Even though the Martell et al. (2011) sample has been selected to belong primarily to the halo field, it is reasonable to expect some contamination from the thick disk and MWTD. However, the prograde features in the rotational velocity distribution may not be simply related to these components. Indeed, a more careful examination of the rotational velocity as a function of $Z_{\max }$ reveals that the highly prograde stars are still present in regions dominated by the halo system, $Z_{\max }>5 \mathrm{kpc}$. These stars are most likely members of substructures; we defer a detailed analysis to a future paper.

We have selected a subsample of metal-poor stars with $[\mathrm{Fe} / \mathrm{H}]<-1.5$ to reduce possible contamination from the thick disk and MWTD, and $Z_{\max }<15 \mathrm{kpc}$ in order to avoid the substructures present in the Martell et al. (2011) data. With these cuts in metallicity and $Z_{\max }$, the total number of stars is $N_{\text {Star }}=$ 360 ; there are $N_{\mathrm{CN}}=10 \mathrm{CN}$-strong stars. Figure 3 (top) shows the Galactocentric rotational velocity distribution of the selected subsample of stars. The left panel represents the low-metallicity $\mathrm{CN}$-normal subsample, while the right panel shows the lowmetallicity $\mathrm{CN}$-strong stars. The mean rotational velocity and dispersion for the CN-normal stars is $\left\langle V_{\phi}\right\rangle=-19 \pm 9 \mathrm{~km} \mathrm{~s}^{-1}$ and $\sigma_{V_{\phi}}=114 \pm 6 \mathrm{~km} \mathrm{~s}^{-1}$, consistent with membership in

\footnotetext{
13 Figure 6 of Carollo et al. (2007), supplemental material.
}

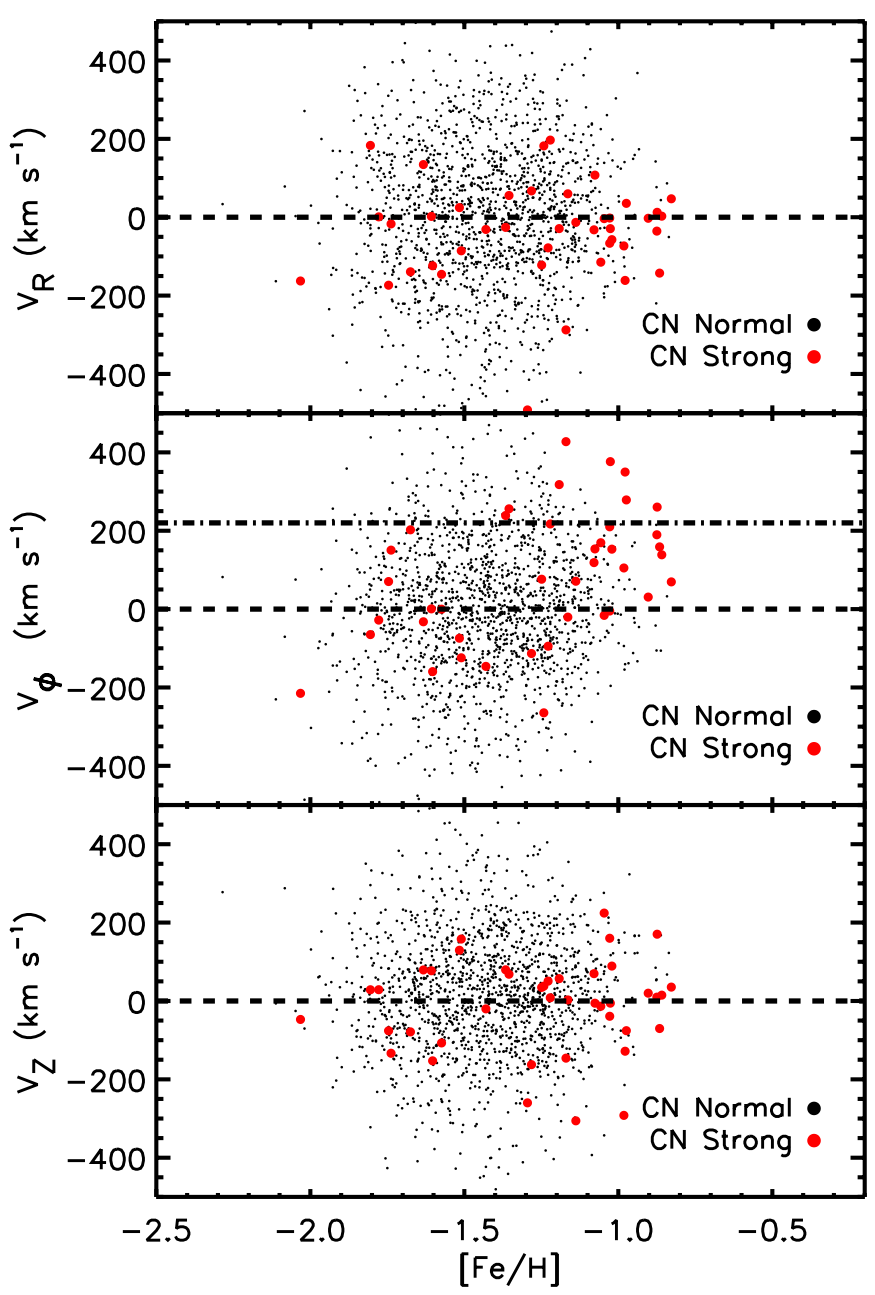

Figure 2. Distribution of the velocity components $\left(V_{R}, V_{\phi}, V_{Z}\right)$ vs. $[\mathrm{Fe} / \mathrm{H}]$ for the stars selected from the Martell et al. (2011) sample. The dot-dashed line in the middle panel is the adopted LSR velocity for stars in the solar neighborhood, while the dashed line in each panel represents the mean velocity of a non-rotating population. The black dots represent the $\mathrm{CN}$-normal stars, while the red dots indicate the $\mathrm{CN}$-strong stars.

(A color version of this figure is available in the online journal.)

the IHP $\left(\left\langle V_{\phi}\right\rangle=7 \pm 4 \mathrm{~km} \mathrm{~s}^{-1}\right.$ and $\sigma_{V_{\phi}}=95 \pm 2 \mathrm{~km} \mathrm{~s}^{-1}$; Carollo et al. 2010). A two-sample Kolmogorov-Smirnov (K-S) test of the distributions of rotational velocity for the low-metallicity $\mathrm{CN}$-normal and $\mathrm{CN}$-strong stars is unable to reject the hypothesis that they were drawn from the same parent population $(p=0.34)$. Note that the number of stars with highly retrograde velocities in the low-metallicity subsample is very small, $N_{\text {retr }}=29$ at $V_{\phi}<-100 \mathrm{~km} \mathrm{~s}^{-1}$ and $N_{\text {retr }}=6$ at $V_{\phi}<-200 \mathrm{~km} \mathrm{~s}^{-1}$, respectively. Among these groups of stars, none of them are $\mathrm{CN}$ strong.

Carollo et al. $(2007,2010)$ have shown that the IHP is dominated by high-eccentricity orbits, while the OHP exhibits a much more uniform distribution of eccentricities (see Figure 4 of the supplemental material in Carollo et al. 2007 and Figure 5 of Carollo et al. 2010). We have used the eccentricity parameter to better quantify the connection between the IHP and the $\mathrm{CN}$-strong stars. Figure 3 (bottom panels) shows the eccentricity distribution for the selected subsamples of stars. As before, the left panel represents the low-metallicity $\mathrm{CN}$-normal subsample, while the right panel shows the low-metallicity $\mathrm{CN}$-strong stars. Inspection of these panels reveals that the $\mathrm{CN}$-normal stars at low metallicity are dominated by high-eccentricity orbits 

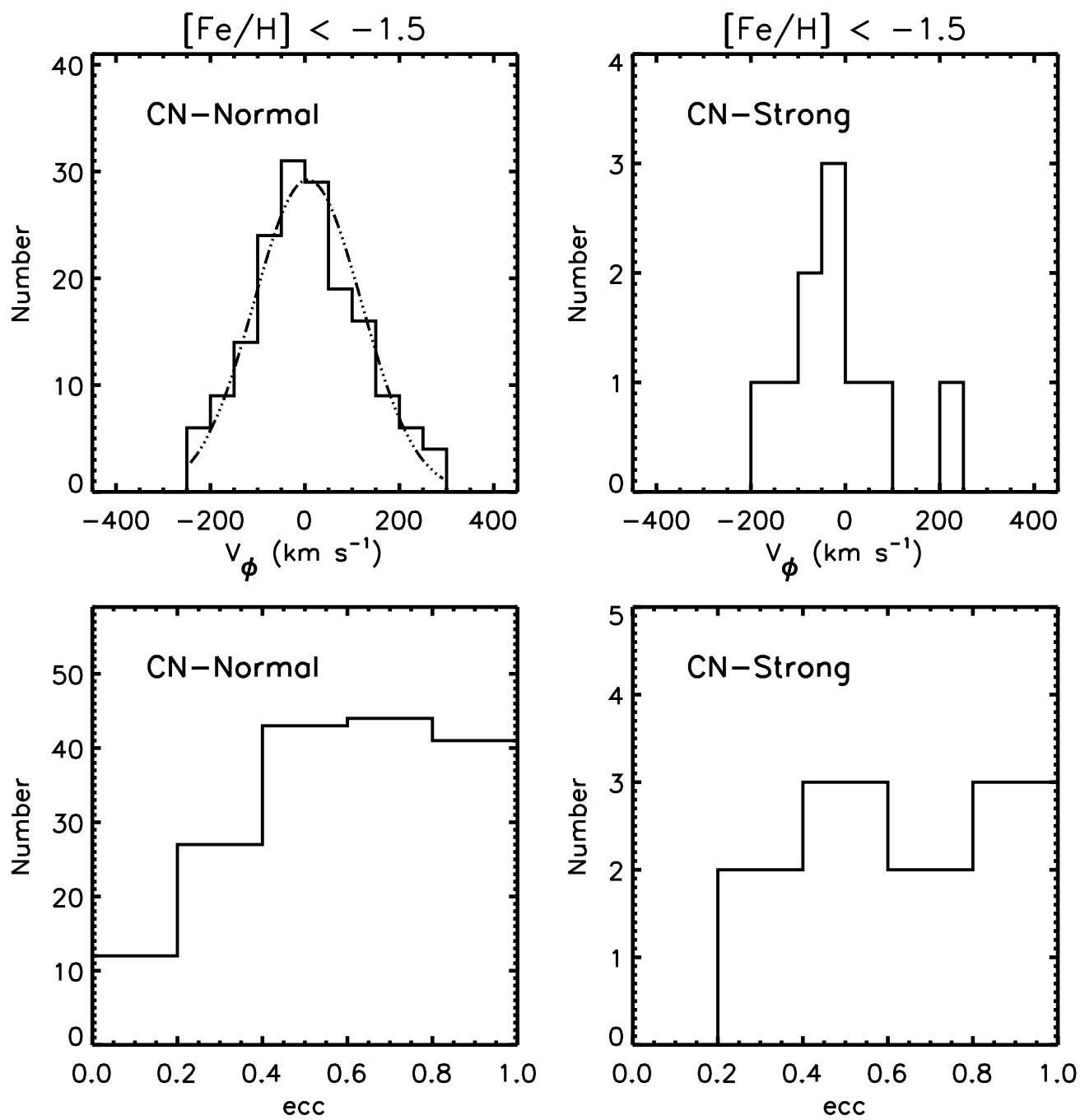

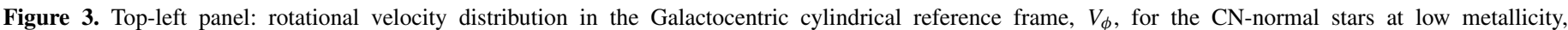

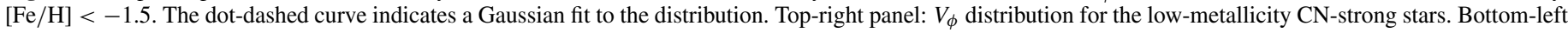

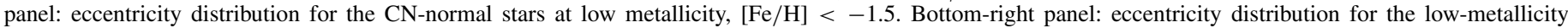
$\mathrm{CN}$-strong stars.

$(e>0.5)$, in agreement with the eccentricity distribution of the IHP. The CN-strong stars are also dominated by higheccentricity orbits. A two-sample K-S test of eccentricity distribution for the low-metallicity $\mathrm{CN}$-normal and $\mathrm{CN}$-strong stars is unable to reject the hypothesis that they were drawn from the same parent population $(p=0.64)$.

\subsection{Comparison with Galactic Globular Clusters}

The general properties of the GCs in our sample with available absolute proper motions are typical of the Milky Way's cluster population in terms of their spatial and metallicity distributions. The metal-rich portion of the sample $([\mathrm{Fe} / \mathrm{H}]>$ $-1.0)$ is concentrated toward the center of the Galaxy, lies close to the Galactic plane, and is rapidly rotating. The metalpoor portion $([\mathrm{Fe} / \mathrm{H}]<-1.5)$ occupies a more spherically symmetric region surrounding the Galactic center and has a slightly prograde mean rotational velocity. The top-left panel of Figure 4 shows the spatial distribution projected onto the $Y Z$ plane in a Galactocentric Cartesian reference system; the red dots denote GCs at low metallicity, $[\mathrm{Fe} / \mathrm{H}]<-1.5$. These GCs are mostly concentrated within $|Z| \sim 15 \mathrm{kpc}$. The top-right panel of Figure 4 shows metallicity for the GCs as a function of
Galactocentric distance. As seen in the figure, the GC sample is primarily located within $0<r<15 \mathrm{kpc}$, and exhibits two metallicity peaks (as previously shown by Zinn 1985) —a small one at $[\mathrm{Fe} / \mathrm{H}] \sim-0.6$ and a dominant one at $[\mathrm{Fe} / \mathrm{H}] \sim-1.6$. The marginal histograms in the right panel show the distribution of $r$ and $[\mathrm{Fe} / \mathrm{H}]$. The bottom panels of Figure 4 show the spatial distribution in the $Y Z$ plane and the metallicity as a function of the Galactocentric distance for the sample of 129 GCs selected from the Harris database, including those with available proper motions. The kinematic and orbital parameters of the GCs with available proper motions are listed in Table 3.

The upper row of panels in Figure 5 shows the rotational velocity distribution ( $V_{\phi}$, Galactocentric cylindrical reference frame) for the entire $\mathrm{GC}_{\mathrm{PM}}$ sample in the left panel, and for the metal-poor subsample $([\mathrm{Fe} / \mathrm{H}]<-1.5)$ in the right panel. In the left panel, the highly prograde feature $\left(V_{\phi} \sim 150-180 \mathrm{~km} \mathrm{~s}^{-1}\right)$ in the velocity distribution is associated with the metal-rich subsample, while the slightly prograde or non-rotating velocity distribution is associated with the metal-poor subsample. The dot-dashed curve in the right panel indicates a Gaussian fit to the distribution, with mean rotational velocity $\left\langle V_{\phi}\right\rangle \sim 1 \mathrm{~km} \mathrm{~s}^{-1}$ and dispersion $\sigma_{V_{\phi}} \sim 136 \mathrm{~km} \mathrm{~s}^{-1}$. These values are consistent 

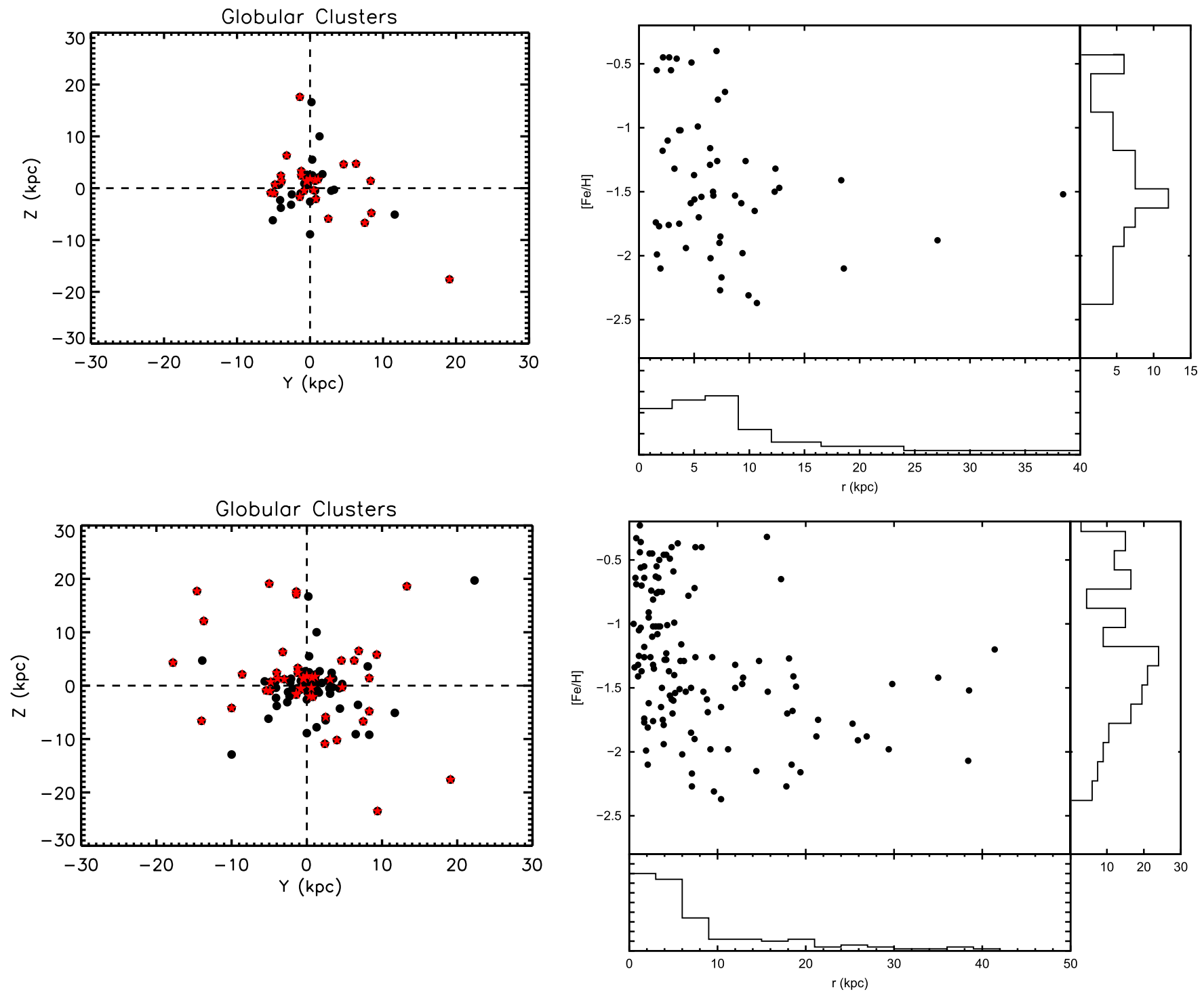

Figure 4. Top-left panel: the distribution of the Galactic globular cluster sample with available absolute proper motions, projected onto the $Y Z$ plane, in the Galactocentric Cartesian reference system, with $(0,0)$ at the Galactic center. The red dots indicate the globular clusters at low metallicity, $[\mathrm{Fe} / \mathrm{H}]<-1.5$. Top-right panel: metallicity as a function of the Galactocentric radius, $r$, for the 59 globular clusters with available absolute proper motions. The marginal histograms denote the distributions of $[\mathrm{Fe} / \mathrm{H}]$ and $r$. Bottom-left panel: the distribution of the 129 Galactic GCs selected from the Harris (1996, 2010 update) database, projected onto the $Y Z$ plane. Bottom-right panel: metallicity as a function of the Galactocentric radius, $r$, for the 129 globular clusters.

(A color version of this figure is available in the online journal.)

with membership in the IHP, perhaps with some contamination from a higher-dispersion population. A two-sample K-S test of the rotational velocity distribution of the low-metallicity GCs and the $\mathrm{CN}$-normal stars is unable to reject the hypothesis that they were drawn from the same parent population $(p=0.5)$. A similar null result is obtained for a two-sample K-S test of the rotational velocity distribution of the low-metallicity GCs and the $\mathrm{CN}$-strong stars $(p=0.2)$.

The lower row of panels in Figure 5 shows the eccentricity distribution for the sample of GCs with no selection in metallicity (left panel), and at low metallicity $[\mathrm{Fe} / \mathrm{H}]<-1.5$ (right panel). In the left panel, the low-eccentricity values are associated with the metal-rich subsample, while the highly eccentric orbits are associated with the metal-poor subsample. In the right panel, the subsample at low metallicity is dominated by higheccentricity orbits, which are typical of the IHP. A two-sample $\mathrm{K}-\mathrm{S}$ test applied to the eccentricity distribution of the metal-poor $\mathrm{GCs}$ and the metal-poor $\mathrm{CN}$-normal stars sample is unable to reject the hypothesis that they were drawn from the same parent population $(p=0.9)$. The same test applied to the eccentricity distribution of the metal-poor GCs and the metal poor $\mathrm{CN}$-strong stars sample is also unable to reject the hypothesis that they were drawn from the same parent population $(p=0.9)$.

We have performed a kinematic analysis for the sample of 129 GCs (comprising the 51 objects with available proper motions and the $78 \mathrm{GCs}$ with no proper motion available) selected from the Harris database, and culled as described in Section 3. Our aim is to explore the rotational properties of a more extended sample of GCs and to check for consistency with the results reported above, which were based on the subset of clusters with available proper motions. We follow the procedure described by Frenk \& White (1980), which makes use of distance and observed radial velocities alone (along with assumed axisymmetry) in order to estimate the rotation and dispersion of Galactic tracer populations. From this approach, the mean rotational velocity derived for the subsample of 
Table 3

Kinematic and Orbital Parameters for the Sample of Globular Clusters with Available Proper Motions

\begin{tabular}{|c|c|c|c|c|c|c|c|c|c|c|c|}
\hline Name & $\begin{array}{c}U \\
\left(\mathrm{~km} \mathrm{~s}^{-1}\right)\end{array}$ & $\begin{array}{c}e_{U} \\
\left(\mathrm{~km} \mathrm{~s}^{-1}\right)\end{array}$ & $\begin{array}{c}V \\
\left(\mathrm{~km} \mathrm{~s}^{-1}\right)\end{array}$ & $\begin{array}{c}e_{V} \\
\left(\mathrm{~km} \mathrm{~s}^{-1}\right)\end{array}$ & $\begin{array}{c}W \\
\left(\mathrm{~km} \mathrm{~s}^{-1}\right)\end{array}$ & $\begin{array}{c}e_{W} \\
\left(\mathrm{~km} \mathrm{~s}^{-1}\right)\end{array}$ & $\begin{array}{c}V_{\phi} \\
\left(\mathrm{km} \mathrm{s}^{-1}\right)\end{array}$ & $\begin{array}{c}e_{V_{\phi}} \\
\left(\mathrm{km} \mathrm{s}^{-1}\right)\end{array}$ & $\begin{array}{l}r_{\max } \\
(\mathrm{kpc})\end{array}$ & $\begin{array}{l}r_{\min } \\
(\mathrm{kpc})\end{array}$ & $\begin{array}{l}Z_{\max } \\
(\mathrm{kpc})\end{array}$ \\
\hline NGC 104 & 84.6 & 10.5 & -75.0 & 11.7 & 47.8 & 5.3 & 166.0 & 6.9 & 7.9 & 6.0 & 3.6 \\
\hline NGC 288 & 26.9 & 11.8 & -293.9 & 33.7 & 51.2 & 0.3 & -74.0 & 33.7 & 12.1 & 3.6 & 9.9 \\
\hline NGC 362 & 25.9 & 25.6 & -296.0 & 29.3 & -68.6 & 24.8 & -37.5 & 24.4 & 10.6 & 1.2 & 7.0 \\
\hline NGC 3201 & -183.0 & 13.7 & -450.7 & 2.7 & 130.4 & 9.0 & -292.2 & 6.7 & 21.9 & 9.2 & 7.4 \\
\hline NGC 4372 & 107.9 & 17.7 & -158.0 & 12.3 & 76.8 & 12.1 & 117.8 & 5.7 & 7.6 & 3.4 & 2.3 \\
\hline NGC 4833 & 95.0 & 24.4 & -291.4 & 15.9 & -42.6 & 10.8 & 23.0 & 18.1 & 8.6 & 0.5 & 1.3 \\
\hline NGC 5024 & -38.3 & 83.9 & 34.7 & 84.6 & -59.8 & 15.1 & 237.3 & 84.3 & 31.5 & 15.8 & 30.2 \\
\hline NGC 5139 & -55.9 & 11.4 & -261.9 & 11.1 & -0.1 & 10.3 & -66.8 & 4.4 & 6.7 & 1.5 & 1.3 \\
\hline NGC 5272 & -64.2 & 26.6 & -111.3 & 25.7 & -135.1 & 5.7 & 118.8 & 26.4 & 16.1 & 5.1 & 13.7 \\
\hline Pal 5 & 78.5 & 14.2 & -333.6 & 39.0 & 15.1 & 13.9 & 111.0 & 39.1 & 18.1 & 9.1 & 17.4 \\
\hline NGC 5897 & 33.5 & 31.5 & -304.9 & 57.3 & 118.6 & 43.8 & 71.3 & 56.3 & 8.8 & 1.9 & 8.1 \\
\hline NGC 5904 & -329.3 & 41.6 & -184.3 & 46.2 & -207.7 & 36.8 & 69.0 & 39.9 & 37.5 & 1.0 & 32.0 \\
\hline NGC 5927 & 207.6 & 15.4 & -116.0 & 22.8 & 36.8 & 15.0 & 232.2 & 3.8 & 6.3 & 4.7 & 0.9 \\
\hline NGC 5986 & 5.4 & 13.7 & -241.2 & 28.3 & 31.5 & 19.4 & 9.6 & 21.6 & 4.5 & 0.2 & 2.4 \\
\hline NGC 6093 & -8.8 & 10.1 & -348.6 & 46.7 & -98.4 & 29.9 & 67.1 & 76.0 & 3.6 & 1.6 & 4.9 \\
\hline NGC 6121 & -49.2 & 2.6 & -235.7 & 21.7 & -10.5 & 4.4 & -18.3 & 21.9 & 6.6 & 0.4 & 0.6 \\
\hline NGC 6144 & -171.5 & 8.5 & -260.4 & 38.0 & 14.4 & 27.8 & -172.1 & 30.2 & 4.1 & 1.4 & 3.2 \\
\hline NGC 6171 & 1.5 & 11.6 & -76.4 & 30.2 & -43.3 & 26.4 & 142.1 & 29.9 & 3.4 & 3.0 & 2.7 \\
\hline NGC 6205 & 227.1 & 28.7 & -77.3 & 21.7 & -137.2 & 20.3 & -30.7 & 28.6 & 21.1 & 5.4 & 21.3 \\
\hline NGC 6218 & -59.2 & 21.8 & -115.4 & 41.3 & -114.4 & 39.8 & 116.3 & 37.7 & 5.4 & 2.9 & 3.3 \\
\hline NGC 6254 & -95.5 & 13.2 & -117.3 & 28.4 & 82.1 & 19.8 & 121.5 & 24.3 & 5.3 & 3.0 & 2.7 \\
\hline NGC 6266 & 79.4 & 3.1 & -68.0 & 14.9 & 71.2 & 14.0 & 170.9 & 11.6 & 3.3 & 1.9 & 1.2 \\
\hline NGC 6273 & -123.5 & 5.7 & -80.7 & 22.7 & 112.4 & 22.2 & -163.2 & 141.4 & 4.2 & 0.5 & 2.6 \\
\hline NGC 6284 & -25.3 & 8.9 & -460.9 & 72.5 & -4.7 & 50.9 & 238.7 & 72.6 & 10.2 & 6.7 & 3.6 \\
\hline NGC 6287 & 281.6 & 7.8 & -212.5 & 40.1 & -8.3 & 36.1 & -15.6 & 45.6 & 8.0 & 0.1 & 3.9 \\
\hline NGC 6293 & 123.5 & 5.8 & -163.8 & 39.2 & -156.1 & 38.9 & -2.5 & 45.4 & 3.5 & 0.0 & 2.6 \\
\hline NGC 6304 & 106.3 & 3.8 & -57.3 & 11.3 & 30.6 & 8.8 & 177.4 & 8.7 & 4.1 & 2.2 & 0.7 \\
\hline NGC 6316 & -69.0 & 9.5 & -166.6 & 33.3 & 36.8 & 29.9 & -69.9 & 36.1 & 2.3 & 1.0 & 1.1 \\
\hline NGC 6333 & -256.2 & 8.3 & -91.9 & 23.3 & -8.2 & 21.1 & 270.3 & 47.4 & 7.7 & 1.1 & 3.1 \\
\hline NGC 6341 & 20.2 & 22.7 & -166.7 & 22.9 & 51.0 & 30.7 & 21.9 & 21.8 & 10.3 & 0.7 & 5.2 \\
\hline NGC 6342 & -150.8 & 6.0 & -234.6 & 38.2 & -9.7 & 27.7 & 144.4 & 52.7 & 2.8 & 0.7 & 1.7 \\
\hline NGC 6356 & -67.2 & 9.9 & -325.0 & 53.4 & 57.3 & 45.2 & 119.2 & 51.8 & 7.3 & 3.3 & 3.1 \\
\hline NGC 6362 & 92.1 & 14.0 & -130.2 & 21.0 & 41.1 & 16.1 & 125.5 & 6.8 & 5.3 & 3.0 & 2.4 \\
\hline NGC 6388 & -34.0 & 8.4 & -200.7 & 29.6 & -21.7 & 21.9 & -38.8 & 18.0 & 2.7 & 0.7 & 1.2 \\
\hline NGC 6397 & 41.3 & 6.0 & -99.4 & 9.7 & -108.7 & 10.3 & 124.9 & 8.5 & 6.6 & 3.4 & 2.2 \\
\hline NGC 6441 & -1.3 & 6.5 & -232.0 & 45.3 & 44.7 & 30.2 & 10.4 & 44.3 & 3.3 & 0.2 & 1.1 \\
\hline NGC 6584 & -70.5 & 26.6 & -371.1 & 52.2 & -184.3 & 41.0 & 54.1 & 64.5 & 13.4 & 1.2 & 9.0 \\
\hline NGC 6626 & -40.1 & 3.4 & -173.6 & 26.8 & -110.0 & 21.4 & 54.5 & 24.0 & 3.2 & 1.0 & 1.2 \\
\hline NGC 6656 & 153.4 & 2.3 & -47.2 & 6.1 & -111.4 & 14.4 & 196.2 & 6.3 & 11.2 & 3.3 & 2.4 \\
\hline NGC 6712 & 99.8 & 5.8 & -35.6 & 11.8 & -136.6 & 20.2 & 34.0 & 39.0 & 7.4 & 0.4 & 2.1 \\
\hline NGC 6723 & 88.8 & 6.6 & -73.6 & 22.2 & 10.4 & 18.2 & 141.5 & 37.6 & 4.0 & 0.2 & 3.6 \\
\hline NGC 6752 & 34.0 & 5.1 & -29.8 & 9.4 & 21.3 & 7.2 & 192.3 & 8.6 & 6.0 & 5.1 & 1.8 \\
\hline NGC 6779 & 108.5 & 40.1 & -80.9 & 21.4 & 3.1 & 44.1 & -33.2 & 32.6 & 12.9 & 0.8 & 1.9 \\
\hline NGC 6809 & -207.9 & 11.1 & -222.6 & 32.4 & -61.4 & 21.4 & 40.3 & 21.4 & 7.3 & 0.7 & 4.5 \\
\hline NGC 6838 & -84.2 & 15.4 & -65.6 & 10.2 & -3.0 & 15.2 & 175.8 & 2.1 & 7.1 & 4.9 & 0.3 \\
\hline NGC 6934 & 69.7 & 62.4 & -533.0 & 54.9 & -120.2 & 74.9 & -54.4 & 74.5 & 40.9 & 6.4 & 39.8 \\
\hline NGC 7006 & -111.8 & 71.4 & -437.2 & 43.4 & 148.1 & 68.0 & 160.9 & 79.5 & 82.9 & 16.9 & 42.7 \\
\hline NGC 7078 & -227.8 & 60.3 & -291.3 & 55.6 & -113.1 & 56.0 & 165.4 & 37.2 & 18.4 & 8.3 & 13.9 \\
\hline NGC 7089 & 100.8 & 42.0 & -215.5 & 42.2 & -328.4 & 51.5 & -92.2 & 40.8 & 35.5 & 6.4 & 34.0 \\
\hline NGC 7099 & 65.2 & 21.2 & -330.2 & 36.8 & 51.0 & 20.1 & -127.6 & 28.2 & 7.1 & 4.2 & 6.2 \\
\hline Pal 13 & 251.4 & 41.1 & -40.0 & 22.2 & -100.7 & 24.6 & -167.7 & 47.8 & 77.4 & 12.1 & 57.0 \\
\hline
\end{tabular}

GCs with Galactocentric distance $r<15 \mathrm{kpc}$ and metallicity $[\mathrm{Fe} / \mathrm{H}]<-1.5(32 \mathrm{GCs})$ is $V_{\text {rot }}=24 \pm 28 \mathrm{~km} \mathrm{~s}^{-1}$, while the dispersion is $\sigma_{\text {los }}=99 \pm 13 \mathrm{~km} \mathrm{~s}^{-1}$. These results are in agreement with the values obtained for the subsample of GCs with available proper motions. Similar results are obtained when the sample at $r<30 \mathrm{kpc}$ and $[\mathrm{Fe} / \mathrm{H}]<-1.5(44 \mathrm{GCs})$ is considered, $V_{\text {rot }}=38 \pm 36 \mathrm{~km} \mathrm{~s}^{-1}$ and $\sigma_{\text {los }}=88 \pm 10 \mathrm{~km} \mathrm{~s}^{-1}$. We conclude that our kinematic results, based on the subsample of GCs with available proper motions, are not unduly biased as a result of this selection.

\section{SUMMARY AND DISCUSSION}

We have analyzed the sample of red giant stars in the halo fields selected by Martell et al. (2011) from the SEGUE-1 and SEGUE-2 surveys, and determined their kinematic and orbital parameters. After removing possible contamination from one or more substructures, mainly found with metallicities above $[\mathrm{Fe} / \mathrm{H}] \sim-1.5$, we have selected a subsample of stars for which we have examined the rotational velocity distribution and the orbits. Also, a sample of GCs with available proper motions 

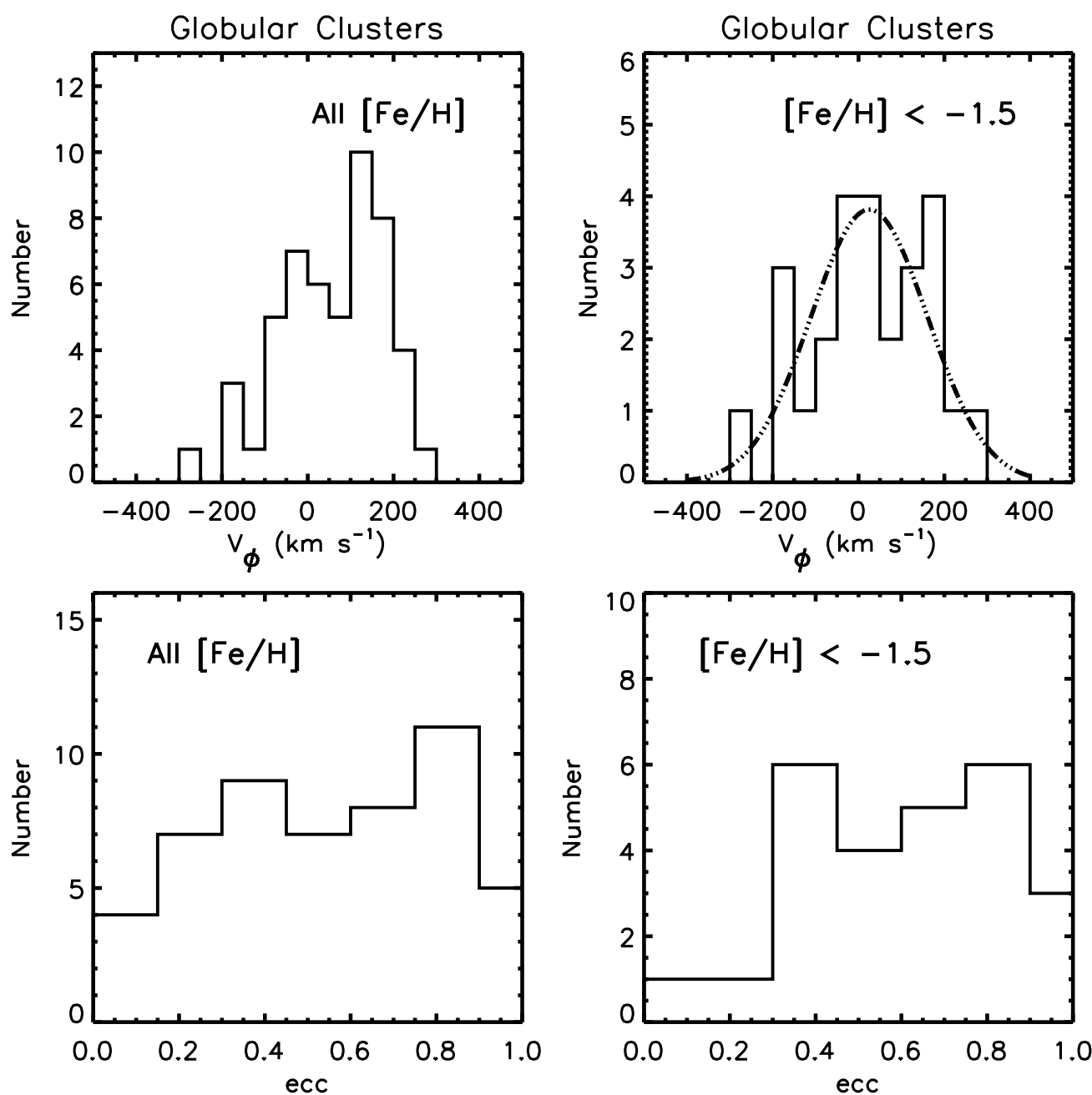

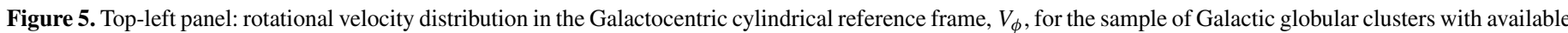

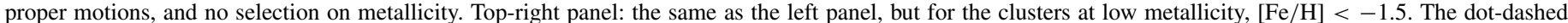

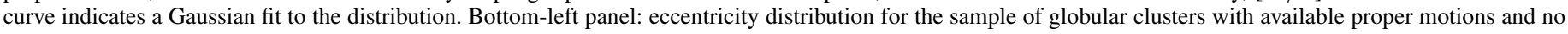
selection on metallicity. Bottom-right panel: the same as in the left panel, but for the clusters at low metallicity, $[\mathrm{Fe} / \mathrm{H}]<-1.5$.

from the literature has been assembled, and its kinematic and orbital properties have been compared to those of the field stars.

Our main results can be summarized as follows.

1. The CN-strong stars are located primarily at Galactocentric radii we associate with the IHR, $r<15-20 \mathrm{kpc}$, where the IHP dominates in the metallicity range covered by this sample.

2. The CN-strong stars occupy orbits that primarily populate $Z_{\max }<15 \mathrm{kpc}$, typical for the orbits of IHP stars.

3. The CN-strong stars exhibit orbits with apogalactic distances below $20 \mathrm{kpc}$, in agreement with the IHP stars.

4. The rotational behavior of the low-metallicity subsample of Martell et al. (2011) $([\mathrm{Fe} / \mathrm{H}]<-1.5)$ is typical of the IHP, with mean velocity and dispersion $\left\langle V_{\phi}\right\rangle=-19 \pm 9 \mathrm{~km} \mathrm{~s}^{-1}$ and $\sigma_{V_{\phi}}=114 \pm 6 \mathrm{~km} \mathrm{~s}^{-1}$, respectively.

5. The eccentricity distribution of the low-metallicity subsample of Martell et al. $(2011)([\mathrm{Fe} / \mathrm{H}]<-1.5)$ is also typical of the IHP, with primarily high-eccentricity orbits.

6. The $\mathrm{CN}$-strong stars in the low-metallicity regime exhibit a rotational velocity distribution consistent with that of the IHP, and which does not differ from the rotational velocity distribution of the low-metallicity $\mathrm{CN}$-normal stars.
7. The $\mathrm{CN}$-strong stars in the low-metallicity regime exhibit an eccentricity distribution consistent with that of the IHP.

8. None of the stars with $\mathrm{CN}$ anomalies possess highly retrograde orbits.

9. The subsample of $\mathrm{CN}$-normal stars at higher metallicity, $[\mathrm{Fe} / \mathrm{H}]>-1.5$, exhibit some evidence for membership in substructures, to be considered in a future paper.

10. The sample of low-metallicity $([\mathrm{Fe} / \mathrm{H}]<-1.5)$ Galactic GCs with available proper motions exhibits a rotational velocity distribution, velocity dispersion, and eccentricity distribution consistent with that of the IHP and the $\mathrm{CN}$-strong stars.

\subsection{Implications for the Formation of the Halo System and the Connection with Galactic Globular Clusters}

Modern high-resolution cosmological simulations at high redshift $(z>3)$ suggest that GCs formed in the central cores of giant, high-density clouds of massive sub-Galactic fragments (or primordial mini-halos; e.g., Bekki 2012). The host progenitor galaxies hierarchically merge onto the main body of the parent galaxy, and are tidally disrupted. However, the GCs that they hosted are sufficiently dense to survive accretion by the main galaxy. In these simulations, most GCs form in 
sub-halos of mass $M \geqslant 10^{9} M_{\odot}$, with masses proportional to the amount of gas present, typically $M_{\mathrm{GC}} \sim 10^{5} M_{\odot}$. These simulations reproduce the distributions of cluster mass, size, and metallicity consistent with those of the Galactic metal-poor clusters (Kravtsov \& Gnedin 2005; Prieto \& Gnedin 2008).

Recent high-resolution simulations of Milky-Way-like galaxies that include prescriptions to account for baryonic material are also able to reproduce the global properties of the inner and outer components of the Milky Way's stellar halo system. In particular, they match well with the observed shift of the stellar MDF toward lower values with increasing Galactocentric distance, and the observed shear in the mean rotational velocity between components (Zolotov et al. 2010; Font et al. 2011; McCarthy et al. 2012; Tissera et al. 2012), as described by Carollo et al. (2007, 2010).

According to the simulations, the IHP is likely to have formed from the rapid dissipational mergers of a number of relatively massive clumps. Star formation within these massive clumps (both pre- and post-merger) would quickly drive up the mean metallicity. The different rotational and orbital properties of stars in the OHP component of the Milky Way clearly indicates that the formation of the outer halo is distinct from that of the inner halo and the disk components, likely through dissipationless accretion of lower-mass subsystems within a pre-existing dark matter halo. A more detailed examination of the nature of the assembly of the IHP and OHP is presented by Tissera et al. (2013).

The fact that the $\mathrm{CN}$-strong stars exhibit spatial distributions, rotational velocities, and orbital properties in agreement with the IHP provides important clues on the origin and fate of GCs in the Milky Way. The primordial sub-Galactic fragments of higher mass and gas content presented favorable conditions to form GCs in the inner cores of giant high-density clouds. In contrast, smaller-mass fragments may not have had sufficient masses of gas to form GCs. These lower-mass mini-halos would likely have had a truncated star-formation history, relative to the higher-mass mini-halos, since they would not have been able to retain gas once star formation commenced. Although further investigation is required, this may account in a natural way for the apparent lack of Galactic GCs with metallicity below $[\mathrm{Fe} / \mathrm{H}] \sim-2.3$. The higher-mass primordial sub-Galactic fragments could be associated with small metal-poor galaxies at high redshift, such as the Ly $\alpha$ emitting galaxies described by Elmegreen et al. (2012). Since it is expected that $\mathrm{CN}$-strong stars require the dense environment of GCs in order to form, their observed properties strongly suggest that a significant fraction of GCs have been stripped or disrupted in the IHR. In this context, the similarity of the global properties of the metal-poor GCs, including their spatial, kinematics, and orbital properties, to those of the $\mathrm{CN}$-strong stars is especially intriguing. Our present data certainly suggest a strong relationship between these two samples - both appear to be associated with the IHP of the Milky Way.

T.C.B. acknowledges partial support from grants PHY 0216783 and PHY 08-22648: Physics Frontier Center/Joint Institute for Nuclear Astrophysics (JINA), awarded by the U.S. National Science Foundation. K.F. acknowledges Sungeun Kim for her advice about the 30 Dor nebula.

Funding for SDSS and SDSS-II has been provided by the Alfred P. Sloan Foundation, the Participating Institutions, the National Science Foundation, the U.S. Department of Energy, the National Aeronautics and Space Administration, the Japanese Monbukagakusho, the Max Planck Society, and the Higher Education Funding Council for England. The SDSS Web site is http://www.sdss.org/.

The SDSS is managed by the Astrophysical Research Consortium for the Participating Institutions. The Participating Institutions are the American Museum of Natural History, Astrophysical Institute Potsdam, University of Basel, University of Cambridge, CaseWestern Reserve University, University of Chicago, Drexel University, Fermilab, the Institute for Advanced Study, the Japan Participation Group, Johns Hopkins University, the Joint Institute for Nuclear Astrophysics, the Kavli Institute for Particle Astrophysics and Cosmology, the Korean Scientist Group, the Chinese Academy of Sciences (LAMOST), Los Alamos National Laboratory, the Max-PlanckInstitute for Astronomy (MPIA), the Max-Planck-Institute for Astrophysics (MPA), New Mexico State University, Ohio State University, University of Pittsburgh, University of Portsmouth, Princeton University, the United States Naval Observatory, and the University of Washington.

Facility: Sloan

\section{REFERENCES}

Abazajian, K. N., Adelman-McCarthy, J. K., Agüeros, M. A., et al. 2009, ApJS, 182,543

Aihara, H., Allende Prieto, C., An, D., et al. 2011, ApJS, 193, 29

Allende Prieto, C., Sivarani, T., Beers, T. C., et al. 2008, AJ, 136, 2070

An, D., Beers, T. C., Johnson, J. A., et al. 2013, ApJ, 763, 65

Baumgardt, H., Kroupa, P., \& Parmentier, G. 2008, MNRAS, 384, 1231

Beers, T. C., Carollo, D., Ivezic, Z., et al. 2012, ApJ, 746, 34

Beers, T. C., \& Christlieb, N. 2005, ARA\&A, 43, 531

Bekki, K. 2012, MNRAS, 421, 44

Bellazzini, M., Ferraro, F. R., \& Ibata, R. 2003, AJ, 125, 188

Bosch, G., Terlevich, E., \& Terlevich, R. 2009, AJ, 137, 3437

Bovy, J., Allende Prieto, C., Beers, T. C., et al. 2012, ApJ, 759, 131

Cannon, R. D., Croke, B. F. W., Bell, R. A., Hesser, J. E., \& Stathakis, R. A. 1998, MNRAS, 298, 601

Carollo, D., Beers, T. C., Bovy, J., et al. 2012, ApJ, 744, 195

Carollo, D., Beers, T. C., Chiba, M., et al. 2010, ApJ, 712, 692

Carollo, D., Beers, T. C., Lee, Y. S., et al. 2007, Natur, 450, 1020

Carretta, E., Bragaglia, A., Gratton, R. G., et al. 2009a, A\&A, 505, 117

Carretta, E., Bragaglia, A., Gratton, R. G., et al. 2009b, A\&A, 505, 139

Carretta, E., Bragaglia, A., Gratton, R. G., et al. 2010, A\&A, 516, A55

Casetti-Dinescu, D. I., Girard, T. M., Korchagin, V. I., van Altena, W. F., \& López, C. E. 2010, AJ, 140, 1282

Cheng, J. Y., Rockosi, C. M., Morrison, H. L., et al. 2012, ApJ, 752, 51

Chiba, M., \& Beers, T. C. 2000, AJ, 119, 2843

Cohen, J. 2004, AJ, 127, 1545

Conroy, C. 2012, ApJ, 758, 21

Cottrell, P., \& Da Costa, G. 1981, ApJL, 245, L79

Dalessandro, E., Schiavon, E., Ricardo, P., et al. 2012, AJ, 144, 126

Decressin, T., Meynet, G., Charbonnel, C., Prantzos, N., \& Ekström, S. 2007, A\&A, 464, 1029

D’Ercole, A., D’Antona, F., Ventura, P., Vesperini, E., \& McMillan, S. L. W. 2010, MNRAS, 407, 854

D’Ercole, A., Vesperini, E., D’Antona, F., McMillan, S. L. W., \& Recchi, S. 2008, MNRAS, 391, 825

de Mink, S. E., Pols, O. R., Langer, N., \& Izzard, R. G. 2009, A\&A, 507, L1 Dinescu, D. I., Girard, T. M., \& van Altena, W. F. 1999, AJ, 117, 1792

Dotter, A., Chaboyer, B., Jevremovic, D., et al. 2008, ApJS, 178, 89 Eisenstein, D. J., Weinberg, D. H., Agol, E., et al. 2011, AJ, 142, 72 Elmegreen, B. G., Malhotra, S., \& Rhoads, J. 2012, ApJ, 757, 9E Font, A. S., McCarthy, I. G., Crain, R. A., et al. 2011, MNRAS, 416, 2802 Forbes, D. A., \& Bridges, T. 2010, MNRAS, 404, 1203

Frebel, A., Christlieb, N., Norris, J. E., et al. 2006, ApJ, 652, 1585

Frenk, C. S., \& White, S. D. M. 1980, MNRAS, 193, 295

Ghez, A. M., Salim, S., Weinberg, N. N., et al. 2008, ApJ, 689, 1044

Gratton, R. G., Bonifacio, P., Bragaglia, A., et al. 2001, A\&A, 369, 87 Gratton, R. G., Sneden, C., \& Carretta, E. 2004, ARA\&A, 42, 385

Gunn, J. E., Siegmund, W. A., Mannery, E. J., et al. 2006, AJ, 131, 2332 Harris, W. E. 1996, AJ, 112, 1487 
Hattori, K., Yoshii, Y., Beers, T. C., Carollo, D., \& Lee, Y. S. 2013, ApJL, 763, L17

Irwin, M. 1999, in IAU Symp. 192, The Stellar Content of Local Group Galaxies, ed. P. Whitelock \& R. Cannon (San Francisco, CA: ASP), 409

Kafle, P. R., Sharma, S., Lewis, G. F., \& Bland-Hawthorn, J. 2013, MNRAS, 430, 2973

Kayser, A., Hilker, M., Grebel, E. K., \& Willemsen, P. G. 2008, A\&A, 486, 437

Kennicutt, R. 1984, ApJ, 287, 116

Kerr, F. J., \& Lynden-Bell, D. 1986, MNRAS, 221, 1023

Kinman, T. D., Cacciari, C., Bragaglia, A., Smart, R., \& Spagna, A. 2012, MNRAS, 422, 2116

Koposov, S. E., Rix, H. W., \& Hogg, D. W. 2009, ApJ, 712, 260

Kraft, R. P. 1994, PASP, 106, 553

Kravtsov, A. V., \& Gnedin, O. Y. 2005, ApJ, 623, 650

Law, D. R., \& Majewski, S. R. 2010, ApJ, 718, 1128

Lee, Y. S., Beers, T. C., Allende Prieto, C., et al. 2011a, AJ, 141, 90

Lee, Y. S., Beers, T. C., An, D., et al. 2011b, ApJ, 738, 187

Lee, Y. S., Beers, T. C., Sivarani, T., et al. 2008a, AJ, 136, 2022

Lee, Y. S., Beers, T. C., Sivarani, T., et al. 2008b, AJ, 136, 2050

Martell, S. L., \& Grebel, E. K. 2010, A\&A, 519, 14

Martell, S. L., Smith, G. H., \& Briley, M. M. 2008, PASP, 120, 839

Martell, S. L., Smolinski, J. P., Beers, T. C., \& Grebel, E. K. 2011, A\&A, 534,136

Massey, P., \& Hunter, D. 1998, ApJ, 493, 180

McCarthy, I. G., Font, A. S., Crain, R. A., et al. 2012, MNRAS, 420, 2245

McLaughlin, D. E., \& Fall, S. M. 2008, ApJ, 679, 1272

Mihalas, D., \& Binney, J. 1981, Galactic Astronomy (San Francisco, CA: Freeman)

Munn, J. A., Monet, D. G., Levine, S. E., et al. 2004, AJ, 127, 3034

Munn, J. A., Monet, D. G., Levine, S. E., et al. 2008, AJ, 136, 895

Nissen, P. E., \& Schuster, W. J. 2010, A\&A, 511, 10

Norris, J. E., Cottrell, P. L., Freeman, K. C., \& Da Costa, G. S. 1981, AJ, 244,205
Palma, C., Majewski, S. R., \& Johnston, K. V. 2002, ApJ, 564, 736

Parmentier, G., Jehin, E., Magain, P., et al. 1999, A\&A, 352, 138

Pilachowski, C. A., Sneden, C., Kraft, R. P., \& Langer, G. E. 1996, AJ, 112,545

Pineda, J. L., Ott, J., Klein, U., Wong, T., \& Muller, E. 2009, ApJ, 703, 736

Prieto, J. L., \& Gnedin, O. Y. 2008, ApJ, 689, 912

Ramirez, I., Meléndez, J., \& Chanamé, J. 2012, ApJ, 757, 164

Ramirez, S. V., \& Cohen, J. D. 2002, AJ, 123, 3277

Rebassa-Mansergas, A., Nebot Gomez-Moran, A., Schreiber, M. R., et al. 2012, MNRAS, 419, 806

Schaerer, D., \& Charbonnel, C. 2011, MNRAS, 413, 2297

Schlegel, D. J., Finkbeiner, D. P., \& Davis, M. 1998, ApJ, 500, 525

Schlesinger, K. J., Johnson, J. A., Rockosi, C. M., et al. 2012, ApJ, 761, 160

Searle, L., \& Zinn, R. 1978, ApJ, 225, 357

Shetrone, M., Martell, S. L., Wilkerson, R., et al. 2010, AJ, 140, 1119

Smolinski, J. P., Lee, Y. S., Beers, T. C., et al. 2011a, AJ, 141, 89

Smolinski, J. P., Martell, S. L., Beers, T. C., \& Lee, Y. S. 2011b, A\&A, 142, 126

Stephens, A., \& Boesgaard, A. M. 2002, AJ, 123, 1647

Tissera, P. B., Scannapieco, C., Beers, T. C., \& Carollo, D. 2013, MNRAS, submitted (arXiv:1301.1301T)

Tissera, P. B., White, S. D. M., \& Scannapieco, C. 2012, MNRAS, 420, 255

Truran, J. W., \& Arnett, W. D. 1971, Ap\&SS, 11, 430

Venn, K. A., Irwin, M., Shetrone, M. D., et al. 2004, AJ, 128, 1177

Ventura, P., D’Antona, F., Mazzitelli, I., \& Gratton, R. 2001, ApJL, 550, L65

Vesperini, E., McMillan, S. L. W., D’Antona, F., \& D’Ercole, A. 2010, ApJL, 718, L112

Vesperini, E., McMillan, S. L. W., D’Antona, F., \& D’Ercole, A. 2013, MNRAS, 429, 1913

Wang, Q. 1999, ApJ, 510, 139

Xue, X., Rix, H. W., Yanny, B., et al. 2011, ApJ, 738, 79

Yanny, B., Rockosi, C. M., Newberg, H. J., et al. 2009, AJ, 137, 4377

York, D. G., Adelman, J., Anderson, J. E., Jr., et al. 2000, AJ, 120, 1579

Zinn, R. 1985, ApJ, 293, 424

Zolotov, A., Willman, B., Brooks, A. M., et al. 2010, ApJ, 721, 738 